\title{
Different presentations of CADASIL; importance of a detailed history
}

\author{
Gayani GGA ${ }^{1}$, Pathirana $\mathrm{KD}^{2}$, Weerarathna $\mathrm{TP}^{2}$, Mohideen $\mathrm{MR}^{2}$ \\ ${ }^{\text {I}}$ Teaching Hospital Karapitiya, Galle, Sri Lanka \\ ${ }^{2}$ Department of Medicine, Faculty of Medicine, University of Ruhuna, Galle, Sri Lanka \\ Correspondence: Dr. G.G.A. Gayani \\ e-mail:aysh2002@gmail.com
}

\section{Introduction}

Cerebral autosomal dominant arteriopathy with subcortical infarcts and leukoencephalopathy (CADASIL) is a rare autosomal dominant arteriopathy characterized by migraine, mood disorders, progressive vascular dementia and recurrent subcortical infarcts. It is caused by mutations in NOTCH3 gene on chromosome 19q12. CADASIL is the most common heritable cause of stroke and vascular dementia in adults with equal sex distribution (1). We report a case of probable CADASIL presented with abnormal behavior.

\section{Case report}

A 33-year-old male admitted with recent change in behavior, aggressiveness and mood swings. There were no specific features to suggest a bipolar affective disorder or depression. He gave a history of migraine for six years which had been managed symptomatically.

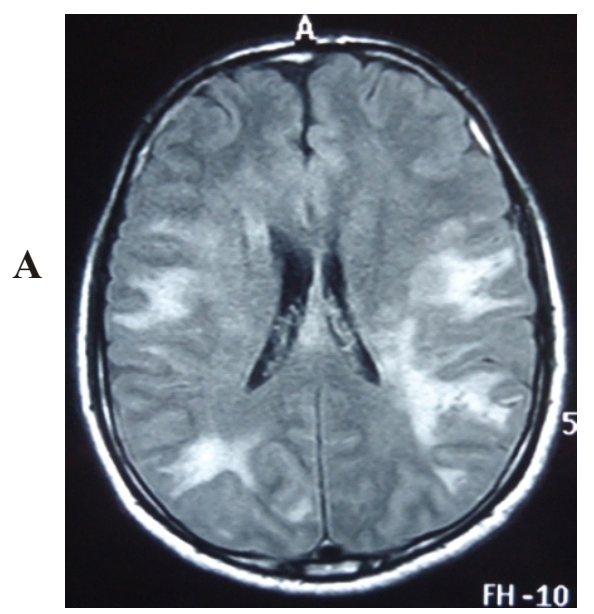

During the previous year he had several transient cerebral ischaemic episodes and minor strokes. One month before admission he has had a similar behavioral change and EEG and Contrast CT Brain done then had been normal. Further questioning revealed slight impairment of his memory. There was no past history of optic neuritis. His mother was on treatment for adult-onset epilepsy since the age of 30 years.

His physical examination was unremarkable including optic fundi. He scored $22 / 30$ on the Montreal Cognitive Assessment (MoCA) test (Normal $\geq 26 / 30$ ) while his mini mental state examination scored $28 / 30$.

MRI of the brain showed T2 weighted hyperintensities on frontal, parietal, temporal and occipital lobes. Lesions were predominantly seen in the subcortical and deep white matter (Figure).

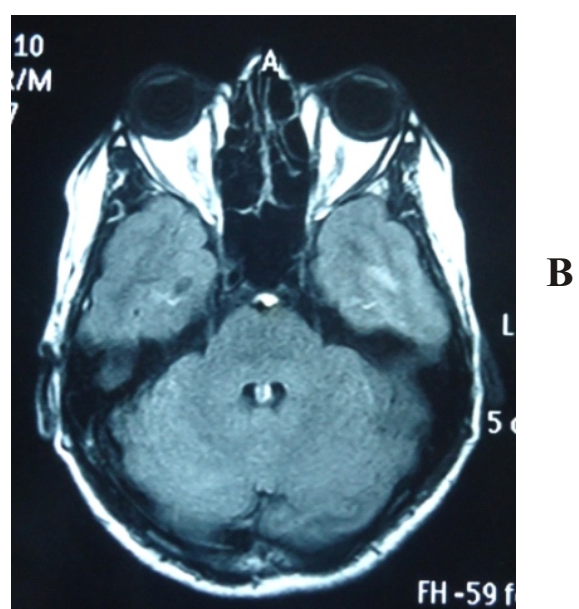

Figure: MRI Brain with FLAIR showing high signal intensity lesions in the periventricular and Subcortical deep white matter (A) High signal intensity lesions in the temporal poles 
Routine haematological and biochemical tests were within the normal range and further investigations showed normal thyroid function tests, homocysteine levels, negative TPHA, ANA, and HIV antibody. Cerebral fluid analysis was normal with a negative result for oligoclonal bands.

We treated him with sodium valproate, folic acid, atorvastatin, propranolol and flunarizine. On discharge patient was apparently well and back to complete independent state.

Three months later he was readmitted with an episode of generalized tonic clonic seizure and receptive aphasia. Repeat MRI brain revealed left side tempero-parietal intra cranial haemorrhage in addition to the initial MRI findings. Later the patient improved with rehabilitation and conservative measures.

According to the symptomatology and spectrum of clinical presentation probable diagnosis of CADASIL was considered. Genetic studies required for confirmation of the diagnosis are currently not available in Sri Lanka.

\section{Discussion}

CADASIL was the probable diagnosis in our patient based on his clinical presentation of altered behavior, migraine, transient cerebral ischaemic episodes, cognitive deficits detected on the MoCA test and the suggestive findings in MRI brain.

CADASIL is essentially characterized by main symptoms of migraine with aura, sub cortical ischaemic events, mood disturbances, apathy and cognitive impairment. The clinical features vary in frequency with age and duration of disease. Typical clinical progression of CADASIL tends to occur sequentially; initially with migraine with aura around the age of 30 years and then transient ischaemic episodes, strokes and mood disorders between the ages of 40 - 60 years. Impairment of executive function is the earliest sign of cognitive impairment in CADASIL (2).

MRI is the investigation of choice. It demonstrates wide spread confluent T2 weighted white matter hyperintensities mainly on periventricular area of frontal and temporal lobes and subcortical deep white matter(3). Genetic analysis is the gold standard in diagnosis. Detection of NOTCH3 mutation or by identifying osmiophilic material within the lamina in skin biopsy on electron microscopy aids in diagnosis of CADASIL. But absence of these findings does not necessarily exclude the disease. Genetic analysis was not done in our patient due to the unavailability of this facility in Sri Lanka $(4,5)$.

NOTCH3 codes for transmembrane receptor protein located on the surface of vascular smooth muscle cells surrounding the arteries. Accumulation of pathologic NOTCH3 receptor protein in small and medium sized cerebral arteries is responsible for the pathogenesis and phenotypic presentation of CADASIL. It's a unique non-arteriosclerotic, non amyloid angiopathy involving small arteries and capillaries, which is largely limited to the brain (1).

There is no specific treatment currently available for CADASIL. Antiplatelet might slow down the disease and help to prevent strokes, but there is no proven benefit. Migraine is managed symptomatically but use of triptan is usually contraindicated due to increased risk of stroke. Administration of tPA following stroke in CADASIL is not advisable due to increased risk of microhaemorrhages (1). Several medications have been used to prevent migraine in patients who have frequent and or severe attacks. It includes sodium valproate, topiramate, gabapentin, propanolol and tricyclic antidepressants. Homocysteine levels are elevated in CADASIL and treatment with folic acid is reasonable (1).

We feel that clinicians may miss many cases of CADASIL if the condition is not suspected and necessary investigations are not performed. As a learning point, this case highlights the importance of obtaining a detailed history in young patients presenting with TIA or strokes. General tendency is to inquire about the conventional risk factors of vascular diseases but the history of migraine with aura and behavioral abnormalities in the past may indicate an alternative etiology. This can influence the management of these patients in two ways. In the acute management thrombolytic agents should be withheld if CADASIL is suspected based on the history. Furthermore, MRI should be chosen as the first imaging technique in suspected cases. This may show the changes typical of CADASIL and hence direct the further investigations and management in a different direction. 


\section{References}

1. Hugueschabriat, Anne Joutel, Martin Dichgans et al. CADASIL. Lancet Neural 2009; 8: 643-53.

2. Dichgans M, Mayer M, Uttner I, et al. The phenotypic spectrum of CADASIL: clinical findings in 102 cases. Ann Neurol 1998; 44: 731.

3. O'Sullivan M, Jarosz JM, Martin RJ, et al. MRI hyperintensities of the temporal lobe and external capsule in patients with CADASIL. Neurology 2001; 56: 628.
4. K.R.D De Silva, R. Gamage, Janavi Dunuwille, et al. Cerebral auto-sominal dominant arteriopathy with subcortical infarcts and leukoencephalopathy (CADASIL): A patient from Sri Lanka. Journal of Clinical Neuroscience 2009; 16: 1492-1493.

5. Champika Gamakaranage, Thashi Chang. CADASIL presenting as a change in personality. Journal of the Ceylon College of Physicians 2011; 42:33-34. 